\title{
Structure and molecular mobility of nascent isotactic polypropylene powders
}

\begin{abstract}
Takeshi Yamanobe $^{1}$, Katsunori Ueki ${ }^{1}$, Tadashi Komoto ${ }^{2,3}$ and Hiroki Uehara ${ }^{1}$
The detailed structure and molecular mobility of nascent isotactic polypropylene (iPP) powder was investigated using differential scanning calorimetry, transmission electron microscopy (TEM) and nuclear magnetic resonance (NMR). The crystallinity of the nascent iPP powder is approximately $33 \%$, which is much lower than that of melt-quenched or isothermally crystallized iPP. Peaks from the variable temperature-cross-polarization and magic-angle spinning spectra at high temperature for the noncrystalline component of nascent powder (POW) were enhanced over those of the melt-crystallized samples. Pulse NMR experiments indicated that the mobility of the rigid component of the POW was easily activated with temperature. No lamellar structures were observed in the TEMs of the POW. The annealing treatment of the POW induced the development of a lamellar structure and temperature independence in the rigid component mobility. From these results, one can conclude that the crystalline size of the POW is extremely small and that the intermediate component is easily affected by the surrounded noncrystalline component.
\end{abstract}

Polymer Journal (2012) 44, 764-771; doi:10.1038/pj.2012.121; published online 20 June 2012

Keywords: iPP; nascent powder; pulse NMR; solid-state NMR

\section{INTRODUCTION}

The morphology of polymers is affected by their crystallization conditions. Crystallization from a dilute solution provides a regular stacking of lamellar crystals, while spherulite structures are produced from a polymer melt. In contrast, crystallization during polymerization forms unique morphologies such as fibrils, whiskers, ribbons and globules. ${ }^{1-4}$ For ultrahigh molecular-weight polyethylene, a cobweb structure composed of particles and fibrils is observed on the surface of the nascent powder (POW). These structures originate from the internal expansion stress during polymerization. The structure of the POW surface depends significantly on the polymerization conditions, especially the polymerization temperature. ${ }^{5-7}$

In addition to a morphological investigation, nuclear magnetic resonance (NMR) studies revealed the phase structure of the POW. ${ }^{8-10}$ The high ductility of the polyethylene POWs as determined by NMR, combined with scanning electron microscope and transmission electron microscopy (TEM), suggested the coexistence of less-entangled noncrystalline phases located between the crystalline and noncrystalline phases. ${ }^{11,12}$ The structural changes during annealing of the polyethylene POWs suggested that the crystalline phases begin to aggregate above the critical temperature, which is $20 \mathrm{~K}$ higher than the polymerization temperature. The NMR and wide-angle X-ray diffraction results reveal that rapid crystallization is repeated following the growth of short-chain segments at the active sites at a low polymerization temperature, whereas the longer chain crystallize at a higher polymerization temperature.
Wristers ${ }^{1}$ used scanning electron microscope to study the effects of catalysts and polymerization conditions on the number and size of fibrils on the surface of nascent isotactic polypropylene (iPP) powder. Kakugo et al..$^{3}$ proposed that the nascent iPP powder is composed of aggregated secondary particles, which are also the aggregation of primary particles. This high-order structure of iPP powder arises from the primary particles, which are developed from catalyst crystallites and disperse uniformly at the initial stage of polymerization within the polymer particles.

The morphology of the nascent iPP powder is primarily investigated on the micrometer level. Loos et al..$^{13}$ analyzed the melting behavior of nascent polyolefins containing iPP with various polymerization conditions and discussed the phase structure of the crystalline and noncrystalline phases taking into account the molecular mobility and the active sites of catalysts on the molecular level. These researchers found that POWs polymerized at low temperature have higher melting temperatures and higher enthalpies of fusion than the melt-crystallized samples, which indicates a stable crystalline phase formation. In contrast, POWs polymerized at high temperatures exhibit a decreased melting temperature and enthalpy of fusion. Ferracini et al. ${ }^{14}$ used WAXS to study the crystalline and noncrystalline phases of nascent iPP powder and concluded that the POW consists of a small number of large crystallites dispersed into a great number of smaller ones that are affected by the structural disorder. These results indicate that the nascent iPP powder polymerized at a high temperature is primarily 
composed of a metastable crystallite. If the crystallite is metastable, the molecular mobility will be easily activated even at temperatures lower than the phase transition temperature such as the melting point. NMR can simultaneously provide information regarding the structure and the molecular mobility for both the crystalline and noncrystalline phases. Combining of NMR with other morphological observation techniques, such as electron microscopy, can provide the detailed structures from the nanoscale to microscale.

This work analyzes the microstructure of nascent iPP powder polymerized at high temperature based on its characterization using differential scanning calorimetry (DSC), TEM and NMR measurements.

\section{EXPERIMENTAL PROCEDURE}

\section{Material}

The iPP POW was supplied by Toho Titanium (Chigasaki, Japan). The iPP was polymerized at $70{ }^{\circ} \mathrm{C}$ using the slurry process with a Ziegler-Natta catalyst (Toho Titanium). The iPP pellets were supplied by Tokuyama. The molecular weights of both iPPs are $500000-600000$

The polymerized iPP was used as the POW sample. The melt-quenched powder sample (PMQ) was prepared by quenching the melted POW sample $\left(210^{\circ} \mathrm{C}\right)$ into an ice-water bath. The iPP pellet was isothermally crystallized (IC) at $135^{\circ} \mathrm{C}$ for $24 \mathrm{~h}$ after melting at $210^{\circ} \mathrm{C}$. The melt-quenched samples (MQs) were prepared by quenching the melted iPP pellet in an ice-water bath. The annealed powder samples were also prepared. The annealing treatments of the POW were carried out at $60,90,110,120$ and $140^{\circ} \mathrm{C}$. The annealed samples are abbreviated as AN followed by the annealing temperature, such as AN60. X-ray analysis confirmed that the crystal structure is $\alpha$-form (monoclinic) for all samples.

\section{Measurements}

DSC. The DSC measurements were carried out using a PerkinElmer Pyris 1 DSC (PerkinElmer, Waltham, MA, USA). The heating run was scanned from 30 to $220^{\circ} \mathrm{C}$ at a heating rate of $20^{\circ} \mathrm{C} \mathrm{min}^{-1}$. All scans were carried out under an $\mathrm{N}_{2}$ atmosphere. The crystallinity of the samples was calculated from the heat of fusion. The enthalpy of fusion for a perfect iPP crystal, $209.2 \mathrm{~J} \mathrm{~g}^{-1}$, was used for the crystallinity estimation.

Solid-state high-resolution ${ }^{13} \mathrm{C}$ NMR. Solid-state high-resolution ${ }^{13} \mathrm{C}$ NMR measurements were carried out on a JEOL EX270W spectrometer (JEOL, Akishima, Japan). A radio frequency of $67.5 \mathrm{MHz}$ was used for the detection of the ${ }^{13} \mathrm{C}$ resonance. The samples were contained in a cylindrical 6-mm zirconia rotor and spun at $5 \mathrm{kHz}$. Cross-polarization and magic-angle spinning (CPMAS) spectra were observed at a temperature range between the room temperature (r.t.) and $100{ }^{\circ} \mathrm{C}$ using a variable temperature (VT) unit with a contact time of $2 \mathrm{~ms}$, a repetition time of $5 \mathrm{~s}$, a $90^{\circ}$ pulse width of $4.5 \mu \mathrm{s}$ and 1024 accumulations. The high-field peak of adamantane was set at 29.5 p.p.m. from tetramethylsilane.

${ }^{1} \mathrm{H}$ pulse NMR. The ${ }^{1} \mathrm{H}$ spin-spin relaxation time $\left(T_{2 H}\right)$ measurements were carried out on a JEOL JNM-MU25 spectrometer using a solid-echo pulse sequence $\left(90^{\circ}-\tau-90^{\circ}\right)$. The $90^{\circ}$ pulse width was $2 \mu$ s. The obtained data were analyzed by fitting with the Weibull and exponential functions (Figure 1).

TEM. The prepared samples were embedded in an epoxy resin, trimmed for microtoming and subsequently stained with $\mathrm{RuO}_{4}$ vapor for $3 \mathrm{~h}$ at $60{ }^{\circ} \mathrm{C}$. Finally, thin sections $(60-\mathrm{nm}$ thick) were prepared at r.t. using a Reichert ULTRACUT S microtome. TEM observations were performed using a JEOL JEM-1200EX electron microscope operated at $80 \mathrm{kV}$.

\section{RESULTS AND DISCUSSION}

The thermal properties and crystallinity of the samples were evaluated by DSC measurement and are summarized in Table 1. Of the samples, POW had the lowest $T_{\mathrm{m}}$ and crystallinity $(33.4 \%)$ of all samples. Because the polymerization temperature was $70^{\circ} \mathrm{C}$, this

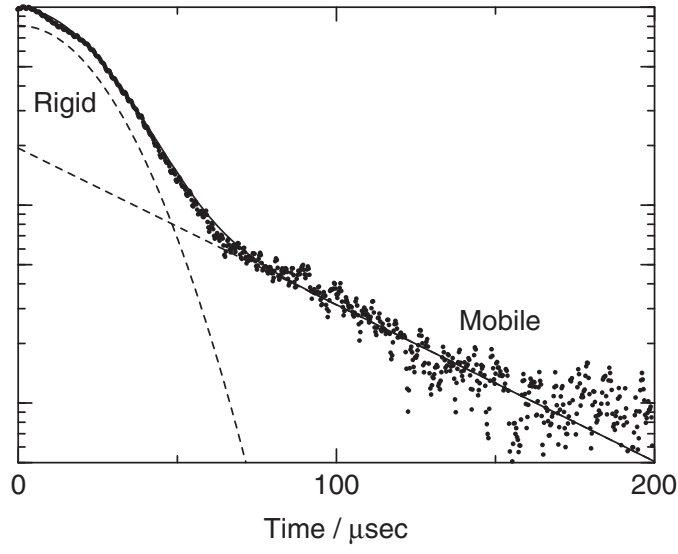

Figure 1 Fitting of FID for POW at r.t.

Table 1 Thermal properties and the crystallinity characterized by DSC

\begin{tabular}{lccc}
\hline Sample & $\mathrm{Tm}\left({ }^{\circ} \mathrm{C}\right)$ & $\Delta H\left(\mathrm{Jg}^{-1}\right)$ & Crystallinity (\%) \\
\hline IC & 174.5 & 132.4 & 63.3 \\
MQ & 167.1 & 91.6 & 43.8 \\
PMQ & 168.9 & 97.6 & 46.7 \\
POW & 163.0 & 69.9 & 33.4 \\
\hline
\end{tabular}

Abbreviations: IC, isothermally crystallized; $M Q$, melt-quenched samples; $P M Q$, melt-quenched powder sample; POW, nascent powder.

result agrees with that reported by Loos et al. ${ }^{13}$ As the crystallinity of POW was lower than that of MQ and PMQ, this structure cannot be achieved via the usual thermal treatment. As noted out by Ferracini et al., ${ }^{14}$ the disordered structure of POW is unique and the crystallization does not appreciably proceed during its polymerization. The melting peak of POW was broader than those of the other samples and had a long tail on the low-temperature side, which implies that the crystalline phase of POW includes a less stable crystallite, which has mobility that is even enhanced at low temperatures. The molecular mobility will be detected by NMR spectroscopy.

Figure 2 shows the ${ }^{13} \mathrm{C}$ CPMAS NMR spectra of POW, PMQ, MQ and IC at r.t. The peaks at approximately 44, 26 and 22 p.p.m. were assigned to the $\mathrm{CH}_{2}, \mathrm{CH}$ and $\mathrm{CH}_{3}$ resonances, respectively. $\mathrm{CH}_{2}$ and $\mathrm{CH}_{3}$ doublets were observed in the spectrum of IC. Because the crystalline form of all samples was the $\alpha$-form as determined by X-ray analysis, doublets of the $\mathrm{CH}_{2}$ and $\mathrm{CH}_{3}$ for IC resulted from the contribution of chain packing in an $\alpha$-form crystal, as reported by Bunn et al. ${ }^{15,16}$ However, the peak splitting of the $\mathrm{CH}_{2}$ and $\mathrm{CH}_{3}$ was not observed for POW, PMQ and MQ. The existence of a disordered state with a 3 -helical conformation has been previously reported. ${ }^{17,18}$ The ${ }^{13} \mathrm{C}$ NMR peak for the disordered state with a $3_{1}$-helical conformation was broad with different relaxation times than the crystalline phase. The crystallinities of POW, PMQ and MQ were lower than that of IC. Therefore, for POW, PMQ and MQ, the contribution of the crystalline component to the ${ }^{13} \mathrm{C}$ NMR peak was low, and the broad disordered state with a $33_{1}$-helix conformation was predominantly reflected in the spectra. Because the doublets of $\mathrm{CH}_{2}$ and $\mathrm{CH}_{3}$ are the direct NMR evidence for local ordering of the packing structure, POW, PMQ and MQ exhibit the packing structure distributions. 


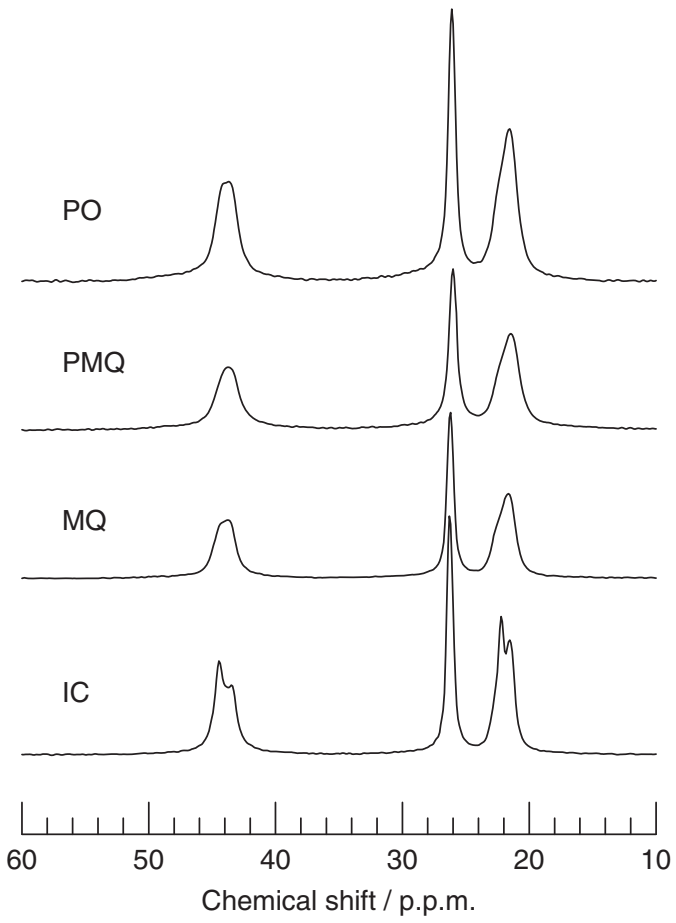

Figure $2{ }^{13} \mathrm{C}$ CPMAS NMR spectra of POW, PMQ, MQ and IC at r.t.

Table $2{ }^{13} \mathrm{C}$ NMR chemical shift and fraction of fitted components for $\mathrm{CH}_{3}$

\begin{tabular}{|c|c|c|c|c|c|c|c|}
\hline \multirow[b]{2}{*}{ Sample } & \multicolumn{3}{|c|}{ Chemical shift/p.p.m. } & \multicolumn{3}{|c|}{ Fraction/\% } & \multirow[b]{2}{*}{ Crystallinity ${ }^{a} / \%$} \\
\hline & 1 & 2 & 3 & 1 & 2 & 3 & \\
\hline IC & 22.8 & 22.2 & 21.5 & 7.1 & 46.6 & 46.3 & 63.3 \\
\hline$M Q$ & 22.5 & 21.7 & 21.2 & 17.4 & 36.6 & 46.1 & 43.8 \\
\hline$P M Q$ & 22.6 & 22.0 & 21.4 & 20.8 & 34.4 & 44.9 & 46.7 \\
\hline POW & 22.5 & 21.8 & 21.3 & 22.5 & 32.5 & 45.0 & 33.4 \\
\hline
\end{tabular}

Abbreviations: IC, isothermally crystallized; $M Q$, melt-quenched samples; $P M Q$, melt-quenched powder sample; POW, nascent powder.

${ }^{a}$ Crystallinities are obtained by differential scanning calorimetry (DSC).

The $\mathrm{CH}_{3}$ peaks of the POW, PMQ and MQ were asymmetrical. In addition to the primary high-field peak, a shoulder peak was observed on the low-field side. For IC, the primary peak split into two peaks and the shoulder peaks, which were clearly observed for POW, PMQ and $\mathrm{MQ}$, were weak at their tails. Careful peak fitting resolved the $\mathrm{CH}_{3}$ peak into three components. The fitted results are summarized in Table 2. The ${ }^{13} \mathrm{C}$ NMR chemical shifts of the three components were approximately $22.6,22.0$ and 21.3 p.p.m., respectively. The peaks at 22.0 and 21.3 p.p.m. were attributed to the crystalline component. The peak at 22.6 p.p.m. was attributed to the noncrystalline component. This assignment can be verified from the behavior of the component fraction. The fraction of the peak at 22.6 p.p.m. increased with a decrease in the crystallinity as determined by DSC. The shape of the $\mathrm{CH}_{3}$ peak for the disordered state was broad and had a tail component at a lower field than the crystalline component. ${ }^{18}$ This low-field tail appeared as a shoulder peak for $\mathrm{CH}_{3}$. In addition, the peak position for the disordered state of $\mathrm{CH}_{3}$ with a $33_{1}$-helix might overlap the noncrystalline component because the peak corresponding to the noncrystalline component did not appear,

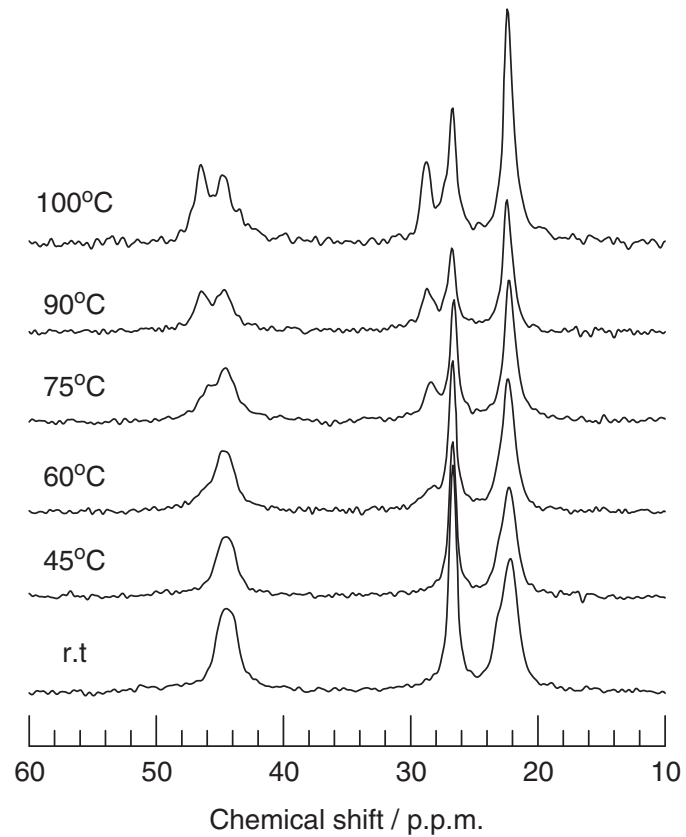

Figure 3 Temperature-dependent ${ }^{13} \mathrm{C}$ CPMAS NMR spectra of POW between r.t. and $100^{\circ} \mathrm{C}$.

even at high temperatures. Therefore, this shoulder component can be used as a measure of the noncrystalline component.

The VT-CPMAS NMR measurements were carried out above r.t. to investigate the temperature dependence of the molecular mobility. Figure 3 shows the temperature dependence of the ${ }^{13} \mathrm{C}$ VT-CPMAS spectrum for POW. Above $60^{\circ} \mathrm{C}$, the low-field peaks can be clearly observed for $\mathrm{CH}_{2}$ and $\mathrm{CH}$. Saito et al. ${ }^{19}$ assigned these peaks to the noncrystalline component in which the polymer chain assumes a liquid-like conformation because the chemical shifts are close to the spectrum of the solution. Such noncrystalline peaks were also confirmed for the other samples. In the spectra obtained at r.t., noncrystalline peaks could not be confirmed due to the restricted molecular motion in the noncrystalline component. These noncrystalline peaks became stronger with increasing temperature, which corresponded to an enhancement in the molecular mobility of the noncrystalline component at elevated temperatures. In contrast, the noncrystalline peak for $\mathrm{CH}_{3}$, which is the low-field shoulder peak, decreased with temperature. At $100{ }^{\circ} \mathrm{C}$, the $\mathrm{CH}_{3}$ peaks were symmetrical and the shoulder peak disappeared completely. Because the molecular motion of the $\mathrm{CH}_{3}$ in the noncrystalline component is fast, the dipolar interaction between $\mathrm{C}$ and $\mathrm{H}$ becomes weak and cross-polarization does not take place effectively at elevated temperatures. Thus, because the ${ }^{13} \mathrm{C}$ CPMAS peaks for both the crystalline and noncrystalline components were observed for the main-chain carbons, $\mathrm{CH}_{2}$ and $\mathrm{CH}$, at elevated temperatures, the static and dynamic structures could be compared between the samples.

Figure 4 provides the CPMAS spectra for POW, PMQ, MQ and IC at $100{ }^{\circ} \mathrm{C}$. At this temperature, the noncrystalline peaks for all samples were recognized on the low-field side of the crystalline peaks for $\mathrm{CH}_{2}$ and $\mathrm{CH}$. The noncrystalline peaks of POW are stronger than the others. Because the crystallinity of POW was lowest of the samples, the quantity of the noncrystalline component should be highest for the POW. However, the peak area of the noncrystalline component of POW was higher than that expected from its crystallinity. The peak 


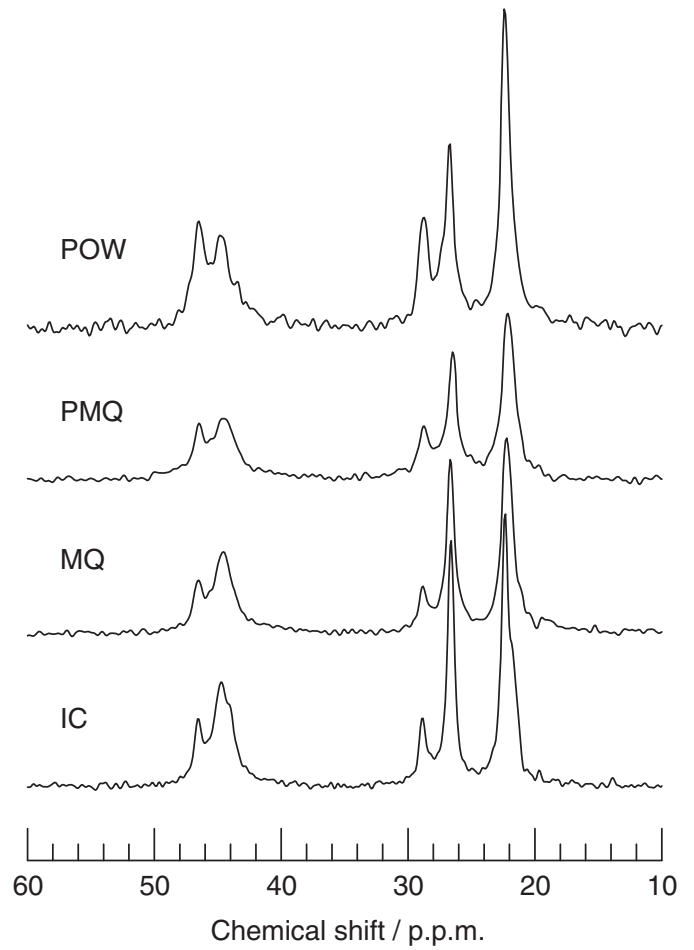

Figure $4{ }^{13} \mathrm{C} C P M A S$ NMR spectra of $P O W, P M Q, M Q$ and $I C$ at $100^{\circ} \mathrm{C}$.

area of the ${ }^{13} \mathrm{C}$ CPMAS depends not only on the quantity but also on the molecular mobility. This suggests that the molecular mobility of POW is different from the others. This trend is understood in two ways: the area of the noncrystalline component for POW is high or the area of the crystalline component for POW is low. The peak area of the CPMAS spectrum is related to the molecular mobility. The noncrystalline peaks for $\mathrm{CH}_{2}$ and $\mathrm{CH}$ are broadened at r.t. because the molecular motion in the noncrystalline component is close to the decoupling frequency. ${ }^{20-22}$ This property was confirmed by the spectra measured at low temperatures because the molecular motion was slower than the decoupling frequency and the decoupling works effectively. VT-CPMAS NMR measurements were carried out at low temperature to confirm this behavior. Figure 5 shows the temperature dependence of the ${ }^{13} \mathrm{C}$ VT-CPMAS spectrum for POW between r.t. and $-60^{\circ} \mathrm{C}$. As the temperature decreased, broad components appeared at the low-field side of the crystalline peaks for $\mathrm{CH}_{2}$ and $\mathrm{CH}$. In addition, a low-field component existed for $\mathrm{CH}_{3}$. Figure 6 shows the expanded and peak-fitted spectra for $\mathrm{CH}_{2}$ at r.t. and $-60{ }^{\circ} \mathrm{C}$. Comparing Figure $6 \mathrm{a}$ with $\mathrm{b}$, the existence of a lowfield component corresponding to an amorphous structure was apparently identified at $-60{ }^{\circ} \mathrm{C}$. Therefore, broad noncrystalline peaks for $\mathrm{CH}_{2}$ and $\mathrm{CH}$ appeared on the low-field side of the crystalline component at $-60^{\circ} \mathrm{C}$. As the temperature increases, those peaks disappeared. The reappearance of the noncrystalline peaks at elevated temperatures indicated that the frequency of the molecular motion became higher than the decoupling frequency. Further increases in the molecular motion caused the peak to diminish. If the molecular motion is excessively rapid, the dipolar interaction between $\mathrm{C}$ and $\mathrm{H}$ becomes too weak for cross-polarization, as observed in the $\mathrm{CH}_{3}$ peak of the noncrystalline component at high temperature. The dependence of the peak height on the molecular mobility can be described as follows. (1) The peak height decreases

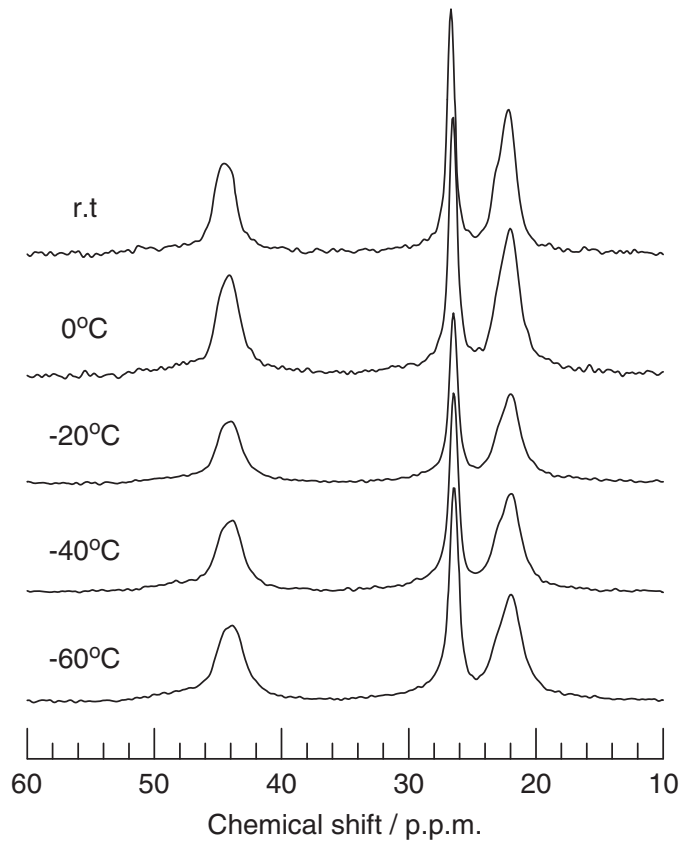

Figure 5 Temperature-dependent ${ }^{13} \mathrm{C}$ CPMAS NMR spectra of POW between r.t. and $-60^{\circ} \mathrm{C}$.
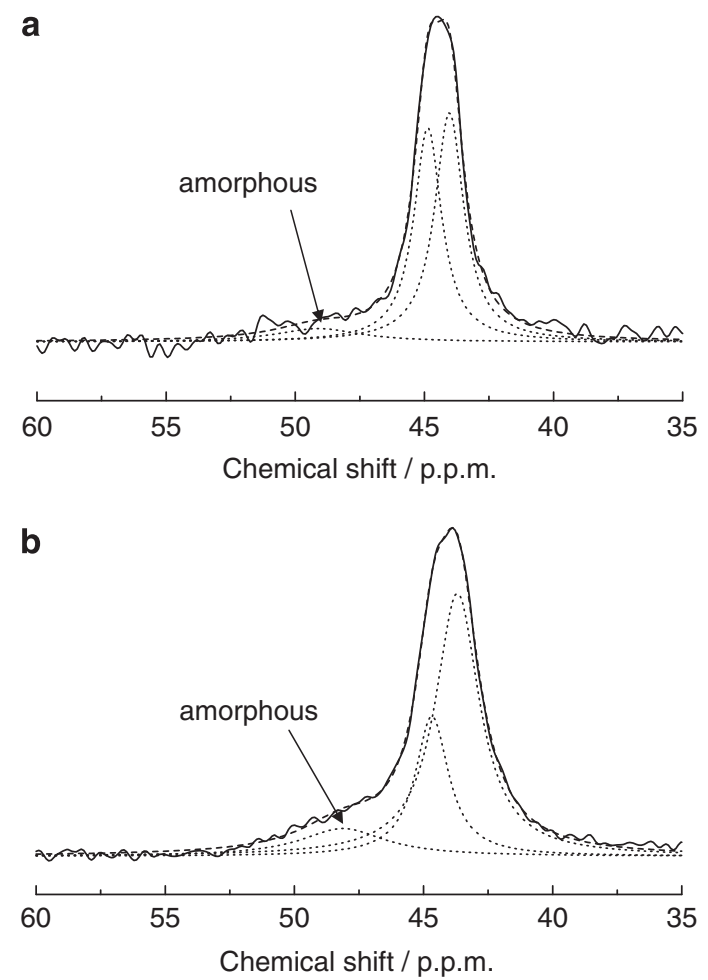

Figure 6 Peak-fitted spectra for the $\mathrm{CH}_{2}$ of POW at (a) r.t. and (b) $-60{ }^{\circ} \mathrm{C}$.

with the initiation of molecular motion. (2) The peak nearly vanishes if the frequency of the molecular motion is close to the decoupling frequency. (3) Further increase in the molecular motion causes the reappearance of the peak and the peak height increases to its maximum value. Taking into account this peak height behavior, for 

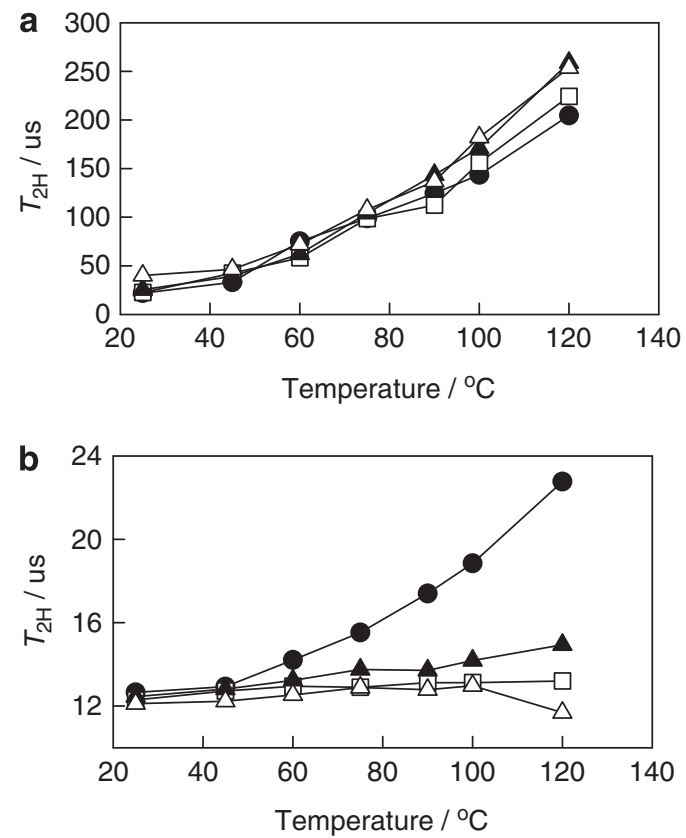

Figure 7 Temperature dependence of the spin-spin relaxation time $\left(T_{2 H}\right)$ of the mobile (a) and rigid (b) components of (-) POW, ( $\mathbf{\Delta}) \mathrm{PMQ},(\square) \mathrm{MQ}$ and $(\Delta)$ IC.

POW, the first possibility indicates that the molecular mobility of the noncrystalline component is higher than that of the others. The second possibility indicates that the molecular mobility of the crystalline component is higher. To decide which possibility is suitable in this case, a discussion of the relaxation time is necessary.

For POW, PMQ, MQ and IC at a temperature range between r.t. and $120{ }^{\circ} \mathrm{C},{ }^{1} \mathrm{H}$ pulse NMR measurements with solid-echo pulse sequence were carried out. The obtained free induction decays (FIDs) were analyzed with the sum of the Weibull and exponential functions. As observed in Figure 1, the FID was well reproduced by these functions. For the component with the longer $T_{2 H}$ (the mobile component), the exponential function was used for the entire temperature range. For the component with the short $T_{2 H}$ (the rigid component), the Weibull coefficient was 2 at r.t. and decreased from 2 to 1 with increasing temperature. In Figures $7 \mathrm{a}$ and $\mathrm{b}$, the temperature dependence of the ${ }^{1} \mathrm{H}$ spin-spin relaxation time $\left(T_{2 H}\right)$ obtained by pulse NMR measurement is shown for the mobile and rigid components, respectively. In Figure $7 \mathrm{a}$, for all samples, the $T_{2 H}$ values for the mobile components increased similarly with temperature, and the temperature dependence of the mobility for mobile components was the same. This result corresponded to the similar enlargement of the noncrystalline peaks for $\mathrm{CH}_{2}$ and $\mathrm{CH}$ resonance in the VT-CPMAS spectra, indicating that the molecular mobility of the noncrystalline component was activated to a similar degree for all samples.

In Figure $7 \mathrm{~b}$, the temperature dependence of the $T_{2 H}$ for the rigid component of POW is clearly different from the others and increases remarkably with temperature. In contrast, the crystalline $T_{2 H}$ of the rigid components for PMQ, MQ and IC are nearly constant and/or increase slightly with temperature. These results indicate that the mobility of the rigid component of POW was easily enhanced with increasing temperature compared with the others. Table 3 summarizes the fractions for the mobile and rigid components. For IC, at r.t., the fraction for the mobile component was 0.1, the lowest among the
Table 3 Temperature dependence of $T_{2}$ fractions

\begin{tabular}{|c|c|c|c|c|c|c|c|c|}
\hline \multirow[b]{2}{*}{ Temperature } & \multicolumn{2}{|c|}{ POW } & \multicolumn{2}{|c|}{$M Q$} & \multicolumn{2}{|c|}{$P M Q$} & \multicolumn{2}{|c|}{ IC } \\
\hline & Mobile & Rigid & Mobile & Rigid & Mobile & Rigid & Mobile & Rig \\
\hline r.t. & 0.19 & 0.81 & 0.22 & 0.78 & 0.19 & 0.81 & 0.10 & 0.90 \\
\hline 45 & 0.28 & 0.72 & 0.26 & 0.74 & 0.24 & 0.76 & 0.22 & 0.79 \\
\hline 60 & 0.20 & 0.80 & 0.23 & 0.77 & 0.24 & 0.76 & 0.24 & 0.76 \\
\hline 75 & 0.23 & 0.77 & 0.23 & 0.78 & 0.25 & 0.76 & 0.24 & 0.76 \\
\hline 90 & 0.26 & 0.74 & 0.28 & 0.72 & 0.24 & 0.77 & 0.25 & 0.75 \\
\hline 100 & 0.29 & 0.71 & 0.24 & 0.76 & 0.24 & 0.76 & 0.24 & 0.76 \\
\hline 120 & 0.36 & 0.64 & 0.29 & 0.71 & 0.29 & 0.71 & 0.24 & 0.76 \\
\hline
\end{tabular}

Abbreviations: IC, isothermally crystallized; $M Q$, melt-quenched samples; $P M Q$, melt-quenched powder sample; POW, nascent powder; r.t., room temperature.

samples, and arises from the high crystallinity. In contrast, the fraction of the mobile components for POW, PMQ and MQ were approximately 0.2 . As the crystallinity for these samples was between 0.3 and 0.5 , the rigid component contained the crystalline component and the intermediate component between the noncrystalline and crystalline components. Therefore, the $T_{2 H}$ for the rigid component was observed as the average of the crystalline and the intermediate components. As the temperature increased, the fraction of the mobile component increased for all samples, indicating that the mobility of a portion of the intermediate component was activated. At $120^{\circ} \mathrm{C}$, the fraction of the rigid component for POW was 0.64, which was the lowest of the samples. The $T_{2 H}$ of the rigid component increased with temperature for POW. Because a part of the intermediate component was activated and measured as the mobile component, the fraction of the crystalline component in the rigid component increased. If the $T_{2 H}$ of the crystalline and intermediate components in the rigid component are temperature-independent, the $T_{2 H}$ of the rigid component decreases with temperature because of a decrease in the intermediate component. However, the $T_{2 H}$ of the rigid component of POW increased, indicating that at least the mobility of the intermediate component was easily activated. In contrast, the $T_{2 H}$ of the rigid components for MQ, PMQ and IC were nearly constant and the mobility of those intermediate components was not easily activated compared with POW. Because the crystallinity for MQ, PMQ and IC were high, it is likely that the hindrance of the mobility activation of the rigid component was caused from the restriction of the mobility of the intermediate component by the crystalline component. In contrast, for POW, the crystalline component cannot restrict the mobility of the intermediate component or the crystalline component itself may begin to fluctuate. This result corresponds to the previously described second possibility

The $T_{2 H}$ measurement obviously indicates that molecular mobility of the rigid component for POW was different from the others. Therefore, this difference might be reflected in the morphologies of the samples. Direct TEM observations were carried out, and Figure 8 shows the TEM micrographs for all samples. A typical thick lamellar structure was observed for IC. For PMQ and MQ, the lamellar structures thinner than that of the IC and cross-hatched structures were observed. In contrast, POW exhibited no lamellar structure, indicating that either the crystalline component was too small to be observed by TEM or there were no ordered crystalline components in the POW. The melting peak, as observed by DSC, indicated that a crystalline component exists in the POW. Therefore, the crystalline size may be small or may contain an irregularity, indicating a 

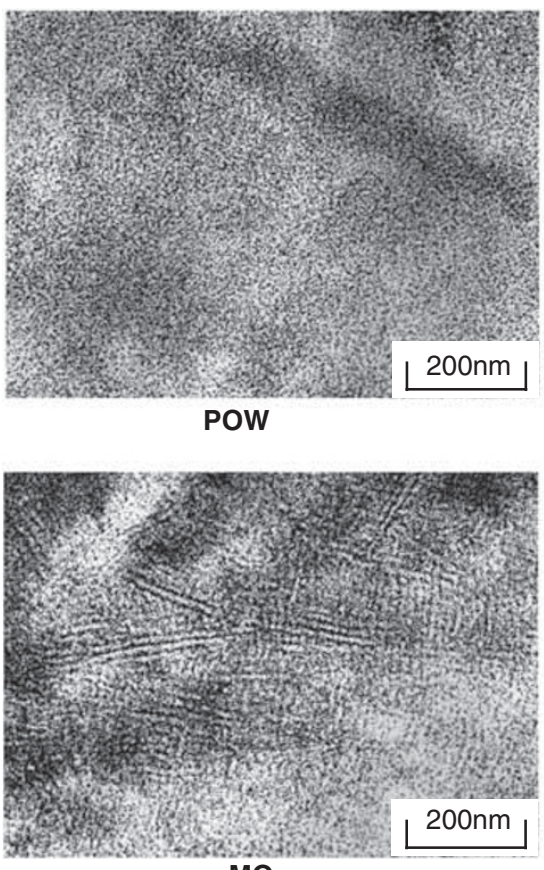

MQ

Figure 8 TEM micrographs of POW, PMQ, MQ and IC.

Table 4 Thermal properties and the crystallinity characterized by DSC

\begin{tabular}{lcccc}
\hline Sample & Annealing temperature $\left({ }^{\circ} \mathrm{C}\right)$ & $\mathrm{Tm}\left({ }^{\circ} \mathrm{C}\right)$ & $\Delta H\left(\mathrm{Jg}^{-1}\right)$ & Crystallinity (\%) \\
\hline POW & Non-annealed & 163.0 & 69.9 & 33.4 \\
AN60 & 60 & 163.0 & 71.1 & 34.0 \\
AN90 & 90 & 163.2 & 81.6 & 39.0 \\
AN110 & 110 & 163.0 & 84.3 & 40.3 \\
AN120 & 120 & 163.0 & 84.8 & 40.5 \\
AN140 & 140 & 162.8 & 94.3 & 45.1 \\
\hline
\end{tabular}

Abbreviation: POW, nascent powder.

remarkable difference between the crystalline sizes of the POW and the others. Considering the results of the pulse NMR measurements, POW exists as a small crystal surrounded by intermediate and noncrystalline components. As the temperature increases, the activation of the mobility of the noncrystalline component induces molecular motion in the intermediate and crystalline components even at low temperatures. Thus, the low crystallinity and temperature dependence of the peak height for POW might arise from its smaller crystalline size.

To confirm the effect of the crystalline size, annealing treatments of the POW were carried out at various temperatures. An increase in the crystalline component upon annealing is generally recognized for crystalline polymers. The thermal properties of the annealed POW, as evaluated by DSC measurements, are summarized in Table 4. The crystallinity increased with the annealing temperature. Figure 9 shows the TEM micrographs of the annealed powders in which a rough lamellar structure was observed in AN110. Lamellae were also clearly observed for AN140. These results confirm that the crystalline size increased and ordered large crystalline components were formed by annealing, thereby corresponding to an increase in crystallinity with annealing temperature.

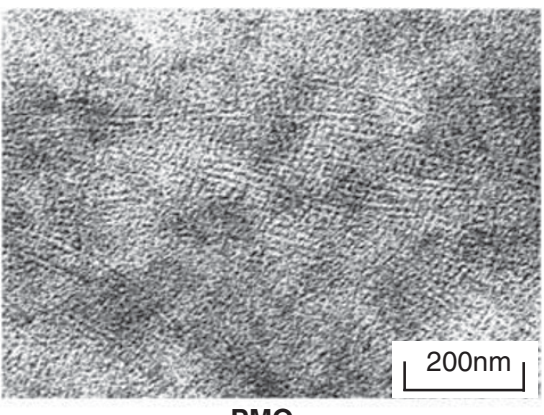

PMQ

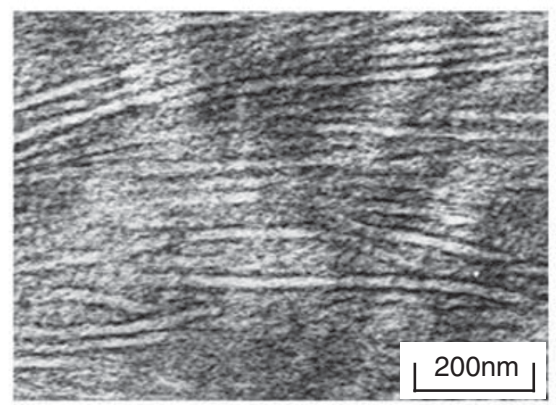

IC

Figure 10a shows the temperature dependence of $T_{2 H}$ for the mobile component of annealed and non-annealed POW. The $T_{2 H}$ of the mobile component exhibited a similar increase with temperature for all samples, which indicates that the molecular mobility of the noncrystalline component increased with temperature to a similar degree. This trend is comparable to that shown in Figure $7 \mathrm{a}$. No differences in molecular mobility were observed for the mobile component of the POW and annealed samples. The temperature dependence of $T_{2 H}$ for the rigid component is shown in Figure 10b. The $T_{2 H}$ value increased rapidly with temperature for POW, AN60 and AN90. For samples annealed at temperatures $>110^{\circ} \mathrm{C}$, the increase in $T_{2 H}$ became moderate and $T_{2 H}$ was nearly constant for AN140, which was similar to the behavior of $T_{2 H}$ for PMQ, MQ and IC that is shown in Figure 7b. Differences in the temperature dependence of $T_{2 H}$ between AN90 and AN110 were attributed to the crystal relaxation ( $\alpha$-relaxation) temperature (approximately $95^{\circ} \mathrm{C}$ ) of iPP. Above the crystalline relaxation temperature, the crystal lattice expanded and the rotation of the polymer chain began, which induced a rearrangement of the crystalline component and a development of the lamellar structure. The temperature dependence of $T_{2 H}$ for the rigid component corresponds to the development of the lamellar structure, that is, a larger lamellar structure has less mobility in the rigid component. From these results, one can conclude that the crystalline size contributes the difference in molecular mobility for the crystalline component.

As a summary of this study, a schematic model of POW is shown in Figure 11. The POW used in this work does not have a clear lamellar structure and possesses only small and thermally unstable crystallites. In Figure 11, the small black squares indicate the crystalline component for POW (upper left). This crystalline component is thermally unstable or contains a structural distribution. At a relatively low temperature, the molecular mobility of the intermediate component is easily activated, and the crystalline component cannot restrict 

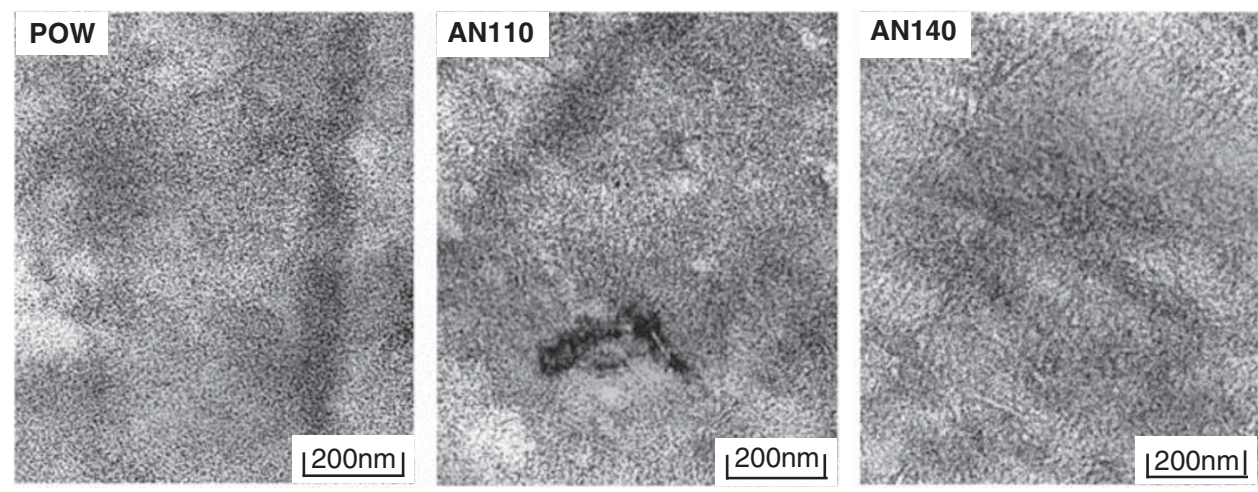

Figure 9 TEM micrographs of non-annealed and annealed powders: POW, AN110 and AN140.
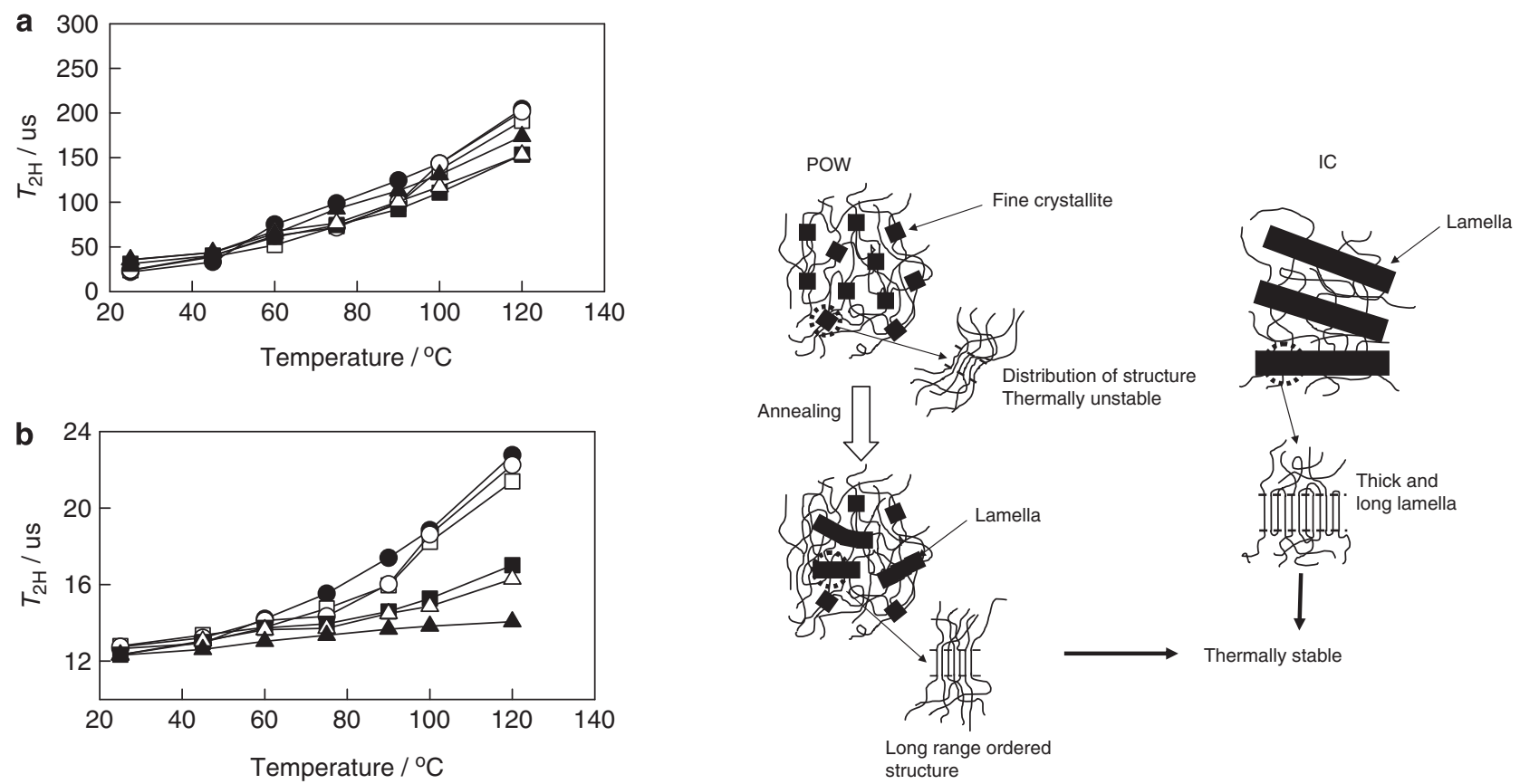

Figure 10 Temperature dependence of the spin-spin relaxation time $\left(T_{2 H}\right)$ of the noncrystalline (a) and crystalline (b) components for the annealed

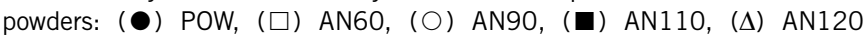
and (ム) AN140.

the mobility of the surrounding intermediate components, as concluded from the pulse NMR and TEM results. Therefore, the crystalline morphology of POW is different from that of PMQ, MQ, IC and annealed samples. Annealing (lower left in Figure 11) at temperature higher than the $\alpha$-relaxation temperature induces a rearrangement of the polymer chain and a development of lamellar structure. Figure 11 shows the development with the annealing of a long-range ordered lamellar structure (a black rectangle) that is thermally stable. Therefore, the developed lamellae can restrict the mobility of the surrounding intermediate components. In addition, the crystalline component becomes thermally stable, and the molecular mobility of the crystalline component is restricted, even at $100^{\circ} \mathrm{C}$. For the IC sample (upper right in Figure 11), a thick and long lamellar structure (the black rectangle) was observed, with the thermal behavior of the molecular mobility begin similar to that of the annealed samples at high temperature. The polymer chains are folded or form an ordered structure with low irregularity. Thus, one

Figure 11 Schematic models of POW, annealed POW and IC.

can conclude that the size of the crystalline component is characteristic to POW and determines its physical properties.

1 Wristers, J. Nascent polypropylene morphology, Polymer fiber. J. Polym. Sci. Polym. Phys. Ed. 11, 1601 (1973).

2 Buls, V. W. \& Higgins, T. L. A particle growth theory for heterogeneous Ziegler polymerization. J. Polym. Sci. 8, 1037 (1970).

3 Kakugo, M., Sadatoshi, H., Sakai, J. \& Yokoyama, M. Growth of polypropylene particles in heterogeneous Ziegler-Natta polymerization. Macromolecules 22, 3172 (1989).

4 Uehara, H., Nakae, M., Kanamoto, T., Ohtsu, O., Sano, A. \& Matsuura, K. Structural characterization of ultrahigh-molecular-weight polyethylene reactor powders based on fuming nitric acid etching. Polymer 39, 6127 (1998).

5 Smith, P., Chanzy, H. D. \& Rotzinger, B. P. Drawing of virgin ultrahigh molecular weight polyethylene: an alternative route to high strength fibres. Polymer Commun. 26, 258 (1985).

6 Smith, P., Chanzy, H. D. \& Rotzinger, B. P. Drawing of virgin ultrahigh molecular weight polyethylene: an alternative route to high strength/high modulus materials. Part 2 influence of polymerization temperature. J. Mater. Sci. 22, 523 (1987). 
7 Rotzinger, B. P., Chanzy, H. D. \& Smith, P. High strength/high modulus polyethylene: synthesis and processing of ultra-high molecular weight virgin powders. Polymer $\mathbf{3 0}$, 1814 (1989).

8 Jarret, W. L., Mathias, L. J. \& Porter, R. S. Solid-state carbon-13 NMR study of polyethylene reactor powders. Macromolecules 23, 5164 (1990).

9 Morin, F. G., Delmas, G. \& Gilson, D. F. R. Variable-temperature solid-state ${ }^{13} \mathrm{C}$ NMR studies of nascent and melt-crystallized polyethylene. Macromolecules 28, 3248 (1995).

10 Gao, P., Cheung, M. K. \& Leung, T. Y. Effects of compaction pressure on cohesive strength and chain mobility of low-temperature compacted nascent UHMWPE. Polymer 37, 3265 (1996).

11 Uehara, H., Yamanobe, T. \& Komoto, T. Relationship between solid-state molecular motion and morphology for ultrahigh molecular weight polyethylene crystallized under different conditions, T. Macromolecules 33, 4861 (2000).

12 Uehara, H., Aoike, T., Yamanobe, T. \& Komoto, T. Solid-State ${ }^{1} \mathrm{H}$ NMR relaxation analysis of ultrahigh molecular weight polyethylene reactor powder. Macromolecules 35, 2640 (2002)

13 Loos, J., Arndt-Rosenau, M., Weingarten, U., Kaminsky, W. \& Lemstra, P. J. Melting behavior of nascent polyolefins synthesized at various polymerization conditions. Polym. Bull. 48, 191 (2002).

14 Ferracini, E., Ferrero, A., Malta, V., Martelli, S. \& Vogel, W. Advanced X-ray study on crystalline and amorphous components of as-polymerized isotactic polypropylene. Colloid Polym. Sci. 269, 1241 (1991).
15 Bunn, A., Cudby, M. E. A., Harris, R. K., Packer, K. J. \& Say, B. J. Solid-state highresolution ${ }^{13} \mathrm{C}$ n.m.r. spectra of polypropene. J. C. S. Chem. Commun. 15 (1981).

16 Bunn, A., Cudby, M. E. A., Harris, R. K., Packer, K. J. \& Say, B. J. High resolution ${ }^{13} \mathrm{C}$ n.m.r. spectra of solid isotactic polypropylene. Polymer 23, 694 (1982).

17 VanderHart, D. L., Alamo, R. G., Nyden, M. R., Kim, M.-H. \& Mandelkern, L. Observation of resonances associated with stereo and regio defects in the crystalline regions of isotactic polypropylene: toward a determination of morphological partitioning. Macromolecules 33, 6078 (2000).

18 Miyoshi, T., Mamun, A. \& Hu, W. Molecular ordering and molecular dynamics in isotactic-polypropylene characterized by solid state NMR. J. Phys. Chem. B. 114, 92 (2010).

19 Saito, S., Moteki, Y., Nakagawa, M., Horii, F. \& Kitamaru, R. High-resolution solid-state carbon-13 NMR study of isotactic polypropylenes isothermally crystallized from the melt. Macromolecules 23, 3256 (1990).

20 Lyerla, J. R. \& Yannoni, C. S. High-resolution carbon-13 NMR of polymers in the solid state. IBM J. Res. Develop. 27, 302 (1983).

21 Takegoshi, K. \& Hikichi, K. Effects of blending on local chain dynamics and glass transition: polystyrene/poly(vinyl methyl ether) blends as studied by high-resolution solid-state ${ }^{13} \mathrm{C}$ nuclear magnetic resonance spectroscopy. J. Chem. Phys. 94, 3200 (1991).

22 Naito, Y., Komoto, T. \& Yamanobe, T. Structure and mobility of a series of poly(alkyl I-glutamate)s studied by VT ${ }^{13}$ C CPMAS NMR spectroscopy. J. Mol. Struct. 602-603, 437 (2002) 